\title{
Degenerate elliptic boundary value problems with asymptotically linear nonlinearity
}

\author{
Kazuaki Taira \\ Dedicated to Professor Hiroshi Fujita on the occasion of his 80th birthday
}

Received: date / Accepted: date

\begin{abstract}
The purpose of this paper is to study a class of semilinear degenerate elliptic boundary value problems with asymptotically linear nonlinearity which include as particular cases the Dirichlet and Robin problems. Our approach is based on the global inversion theorems between Banach spaces, and is distinguished by the extensive use of the ideas and techniques characteristic of the recent developments in the theory of partial differential equations. By making use of the variational method, we prove existence and uniqueness theorems for our problem. The results here extend three earlier theorems due to Ambrosetti and Prodi to the degenerate case.
\end{abstract}

Keywords Semilinear boundary value problem - degenerate boundary condition . asymptotically linear nonlinearity $\cdot$ global inversion theorem $\cdot$ variational method

Mathematics Subject Classification (2010) $35 \mathrm{~J} 65 \cdot 35 \mathrm{~J} 25 \cdot 47 \mathrm{H} 10$

\section{Statement of main results}

Let $\Omega$ be a bounded domain of Euclidean space $\mathbf{R}^{N}, N \geq 2$, with smooth boundary $\partial \Omega$; its closure $\bar{\Omega}=\Omega \cup \partial \Omega$ is an $N$-dimensional, compact smooth manifold with boundary. Let $A$ be a second-order, elliptic differential operator with real coefficients such that

$$
A u=-\sum_{i=1}^{N} \frac{\partial}{\partial x_{i}}\left(\sum_{j=1}^{N} a^{i j}(x) \frac{\partial u}{\partial x_{j}}\right)+c(x) u
$$

Here:

Kazuaki Taira

Institute of Mathematics, University of Tsukuba, Tsukuba 305-8571, Japan

E-mail: taira@math.tsukuba.ac.jp 
(1) $a^{i j} \in C^{\infty}(\bar{\Omega})$ and $a^{i j}(x)=a^{j i}(x)$ for all $x \in \bar{\Omega}$ and $1 \leq i, j \leq N$, and there exists a positive constant $a_{0}$ such that

$$
\sum_{i, j=1}^{N} a^{i j}(x) \xi_{i} \xi_{j} \geq a_{0}|\xi|^{2} \quad \text { for all }(x, \xi) \in \bar{\Omega} \times \mathbf{R}^{N} .
$$

(2) $c \in C^{\infty}(\bar{\Omega})$ and $c(x) \geq 0$ in $\Omega$.

Let $B$ be a first-order, boundary condition with real coefficients such that

$$
B u=a\left(x^{\prime}\right) \frac{\partial u}{\partial v}+b\left(x^{\prime}\right) u
$$

Here:

(3) $a \in C^{\infty}(\partial \Omega)$ and $a\left(x^{\prime}\right) \geq 0$ on $\partial \Omega$.

(4) $b \in C^{\infty}(\partial \Omega)$ and $b\left(x^{\prime}\right) \geq 0$ on $\partial \Omega$.

(5) $\partial / \partial v$ is the conormal derivative associated with the operator $A$ :

$$
\frac{\partial}{\partial v}=\sum_{i, j=1}^{N} a^{i j}\left(x^{\prime}\right) n_{j} \frac{\partial}{\partial x_{i}}
$$

where $\mathbf{n}=\left(n_{1}, n_{2}, \ldots, n_{N}\right)$ is the unit exterior normal to the boundary $\partial \Omega$.

Our fundamental hypotheses on the boundary condition $B$ are the following:

(H.1) $a\left(x^{\prime}\right)+b\left(x^{\prime}\right)>0$ on $\partial \Omega$.

(H.2) $b\left(x^{\prime}\right) \not \equiv 0$ on $\partial \Omega$.

It should be noticed that if $a\left(x^{\prime}\right) \equiv 0$ and $b\left(x^{\prime}\right) \equiv 1$ on $\partial \Omega$ (resp. $a\left(x^{\prime}\right) \equiv 1$ on $\partial \Omega$ ), then the boundary condition $B$ is the Dirichlet condition (resp. Robin condition). Moreover, it is easy to see that boundary condition $B$ is non-degenerate (or coercive) if and only if either $a\left(x^{\prime}\right)>0$ on $\partial \Omega$ or $a\left(x^{\prime}\right) \equiv 0$ and $b\left(x^{\prime}\right)>0$ on $\partial \Omega$. Therefore, our boundary condition $B$ is a degenerate boundary value problem from an analytical point of view (cf. [12]).

The intuitive meaning of hypothesis (H.1) is that either the reflection phenomenon or the absorption phenomenon does occur at each point of the boundary $\partial \Omega$. More precisely, hypothesis (H.1) implies that the absorption phenomenon occurs at each point of the set $M=\left\{x^{\prime} \in \partial \Omega: a\left(x^{\prime}\right)=0\right\}$, while the reflection phenomenon occurs at each point of the set $\partial \Omega \backslash M=\left\{x^{\prime} \in \partial \Omega: a\left(x^{\prime}\right)>0\right\}$ (see [17]). On the other hand, hypothesis (H.2) implies that the boundary condition $B$ is not equal to the purely Neumann condition.

In this paper we consider the following semilinear elliptic boundary value problem in the framework of Hölder spaces: Let $p(\xi)$ be a function defined on R. Given a function $h(x)$ in $\Omega$, find a function $u(x)$ in $\Omega$ such that

$$
\begin{cases}A u-p(u)=h & \text { in } \Omega, \\ B u=a\left(x^{\prime}\right) \frac{\partial u}{\partial v}+b\left(x^{\prime}\right) u=0 & \text { on } \partial \Omega .\end{cases}
$$


Hammerstein [11] and Dolph [10] studied problem (1.3) under Dirichlet condition (see Remarks 1.1 and 1.2), while Amann [2] studied problem (1.3) in the non-degenerate case where the boundary $\partial \Omega$ is the disjoint union of the two closed subsets $M$ and $\partial \Omega \backslash M$, each of which is an $(N-1)$ dimensional, compact smooth manifold.

The purpose of this paper is to prove existence and uniqueness theorems for problem (1.3), and is a continuation of the previous work Taira [19], [20] and [21].

In order to study problem (1.3) in the framework of Hölder spaces, we consider the linear elliptic boundary value problem

$$
\begin{cases}A u=g & \text { in } \Omega \\ B u=0 & \text { on } \partial \Omega\end{cases}
$$

in the framework of the Hilbert space $L^{2}(\Omega)$. We associate with problem (1.4) a densely defined, closed linear operator

$$
\mathfrak{A}: L^{2}(\Omega) \longrightarrow L^{2}(\Omega)
$$

as follows:

(1) $D(\mathfrak{A})=\left\{u \in W^{2,2}(\Omega): B u=0\right.$ on $\left.\partial \Omega\right\}$.

(2) $\mathfrak{A} u=A u$ for every $u \in D(\mathfrak{A})$.

Here and in the following the Sobolev space $W^{k, p}(\Omega)$ for $k \in \mathbf{N}$ and $1<p<\infty$ is defined as follows:

$$
\begin{gathered}
W^{k, p}(\Omega)=\text { the space of functions } u \in L^{p}(\Omega) \text { whose derivatives } D^{\alpha} u, \\
|\alpha| \leq k, \text { in the sense of distributions are in } L^{p}(\Omega),
\end{gathered}
$$

and its norm $\|\cdot\|_{W^{k, p}(\Omega)}$ is given by the formula

$$
\|u\|_{W^{k, p}(\Omega)}=\left(\sum_{|\alpha| \leq k} \int_{\Omega}\left|D^{\alpha} u(x)\right|^{p} d x\right)^{1 / p} .
$$

Then we have the following fundamental spectral results (i), (ii) and (iii) of the operator $\mathfrak{A}$ (see [18, Theorem 5.1]):

(i) The operator $\mathfrak{A}$ is positive and selfadjoint in $L^{2}(\Omega)$.

(ii) The first eigenvalue $\lambda_{1}$ of $\mathfrak{A}$ is positive and algebraically simple, and its corresponding eigenfunction $\phi_{1} \in C^{2+\alpha}(\bar{\Omega})$, with exponent $0<\alpha<1$, may be chosen to be strictly positive in $\Omega$. Namely, we have the assertions

$$
\begin{cases}A \phi_{1}=\lambda_{1} \phi_{1} & \text { in } \Omega, \\ \phi_{1}>0 & \text { in } \Omega \\ B \phi_{1}=0 & \text { on } \partial \Omega\end{cases}
$$

(iii) No other eigenvalues $\lambda_{j}, j \geq 2$, have positive eigenfunctions.

First, we impose the following three conditions (P.1) through (P.3) on the nonlinear term $p(\xi)$ : 
(P.1) $p \in C^{1+\alpha}(\mathbf{R})$ with $0<\alpha<1$ and $p(\xi) \geq 0$ on $\mathbf{R}$.

(P.2) There exist a positive constant $\gamma<\lambda_{1}$ and a non-negative constant $b$ such that

$$
p(\xi) \leq \gamma \xi+b \text { for all } \xi \geq 0
$$

(P.3) $p^{\prime}(\xi)<\lambda_{1}$ on $\mathbf{R}$.

Example 1.1 A simple example of the nonlinear term $p(\xi)$ is given by the formula

$$
p(\xi)= \begin{cases}\gamma\left(\xi+\frac{1}{2 \xi}-\frac{5}{4}\right) & \text { for } \xi>1 \\ \frac{\gamma}{4} \xi^{2} & \text { for }-1 \leq \xi \leq 1 \\ \gamma\left(-\xi-\frac{1}{2 \xi}-\frac{5}{4}\right) & \text { for } \xi<-1\end{cases}
$$

where $0<\gamma<\lambda_{1}$. This function $p(\xi)$ satisfies condition (P.2) with $b=0$.

We remark that condition (P.2) implies the asymptotically linear nonlinearity of $p(\xi)$, while condition (P.3) guarantees that the range $\left\{p^{\prime}(\xi): \xi \in \mathbf{R}\right\}$ of $p^{\prime}$ does not contain the eigenvalues $\lambda_{k}$ of the operator $\mathfrak{A}$.

The next existence and uniqueness theorem for problem (1.3) is a generalization of Ambrosetti-Prodi [4, Chapter 3, Theorem 1.13] to the degenerate case:

Theorem 1.1 Assume that $p(\xi)$ satisfies conditions (P.1) through (P.3). Then the semilinear elliptic boundary value problem (1.3) has a unique solution $u \in C^{2+\alpha}(\bar{\Omega})$ for any function $h \in C^{\alpha}(\bar{\Omega})$.

Remark 1.1 Hammerstein [11] proved Theorem 1.1 in the Dirichlet case ([11, Satz 6]).

Secondly, we impose the following four conditions (Q.1) through (Q.4) on the nonlinear term $p(\xi)$ :

(Q.1) $p(\xi)=a \xi+q(\xi)$ on $\mathbf{R}$.

(Q.2) $a$ is a real constant such that $a \neq \lambda_{k}$ for all $k=1,2, \ldots$.

(Q.3) $q \in C^{1+\alpha}(\mathbf{R})$ with $0<\alpha<1$ and is bounded on $\mathbf{R}$.

(Q.4) Either $p^{\prime}(\xi)=a+q^{\prime}(\xi)<\lambda_{1}$ on $\mathbf{R}$ or $\lambda_{k}<p^{\prime}(\xi)=a+q^{\prime}(\xi)<\lambda_{k+1}$ on $\mathbf{R}$ for some $k$.

Example 1.2 A simple example of the nonlinear term $q(\xi)$ is given by the formula

$$
q(\xi)=\left(\lambda_{1}-a\right) \frac{\xi}{1+\xi^{2}} .
$$

This function $q(\xi)$ satisfies the condition that $a+q^{\prime}(\xi)<\lambda_{1}$ on $\mathbf{R}$.

We remark that conditions (Q.1), (Q.2) and (Q.3) imply that $p(\xi)$ is a bounded nonlinear perturbation of a linear invertible operator (cf. [16, Subsection 6.3.2]). On the other hand, condition (Q.4) implies that the range $\left\{p^{\prime}(\xi): \xi \in \mathbf{R}\right\}$ of $p^{\prime}=a+q^{\prime}$ does not contain the eigenvalues $\lambda_{k}$ of the operator $\mathfrak{A}$.

The next existence and uniqueness theorem for problem (1.3) is a generalization of Ambrosetti-Prodi [4, Chapter 4, Theorem 1.4] to the degenerate case: 
Theorem 1.2 Assume that $p(\xi)$ satisfies conditions $(Q .1)$ through (Q.4). Then problem (1.3) has a unique solution $u \in C^{2+\alpha}(\bar{\Omega})$ for any $h \in C^{\alpha}(\bar{\Omega})$.

Remark 1.2 Dolph [10] and Castro-Lazer [6] proved Theorem 1.2 in the Dirichlet case ([10, Theorem 3.1]; [6, Theorem D]). On the other hand, Ambrosetti-Prodi [3] considered the case where the range of $p^{\prime}$ contains only the first eigenvalue $\lambda_{1}$ of the Dirichlet problem, and studied problem (1.3) in the framework of singularity theory in Banach spaces ([22]). They characterized completely the solution structure of problem (1.3) ([3, Theorem 3.1], [4, Chapter 4, Theorem 2.4]; see also [5, Theorem $3])$. Their result is generalized to the degenerate case by Taira ([19, Theorem 1.1]).

Thirdly, we replace conditions (Q.3) and (Q.4) by a weaker condition

(Q.5) $q(\xi)$ is bounded and Lipschitz continuous on $\mathbf{R}$.

Example 1.3 A simple example of the nonlinear term $q(\xi)$ is given by the formula

$$
q(\xi)= \begin{cases}a\left(\frac{1}{2 \xi}-1\right) & \text { for } \xi>1, \\ -\frac{a}{2} \xi & \text { for }-1 \leq \xi \leq 1, \\ a\left(\frac{1}{2 \xi}+1\right) & \text { for } \xi<-1\end{cases}
$$

where $a$ is a real constant.

The next existence theorem for problem (1.3) is a generalization of AmbrosettiProdi [4, Chapter 4, Theorem 1.1] to the degenerate case:

Theorem 1.3 Assume that $p(\xi)$ satisfies conditions (Q.1), (Q.2) and (Q.5). Then problem (1.3) is solvable. More precisely, problem (1.3) has a solution $u \in C^{2+\alpha}(\bar{\Omega})$ for any $h \in C^{\alpha}(\bar{\Omega})$.

The rest of this paper is organized as follows. Section 2 deals with local and global inversions of mappings between Banach spaces which go back to Hadamard in the finite dimensional case and to Cacciopoli and Lévy for general Banach spaces (Theorem 2.2). In Section 3 we summarize the basic facts about the Leray-Schauder degree introduced by Leray-Schauder [13] in the study of nonlinear partial differential equations. The non-triviality of the degree guarantees the existence of a solution of nonlinear equations (Theorem 3.1). In Section 4 we prove Theorem 1.1, by using global inversion theorems, just as in Ambrosetti-Prodi [4, Theorem 1.13]. To do this, we have only to verify all the conditions of Theorem 2.2. Our proof of Theorem 1.1 is based on the variational approach developed in Taira [18] and [19] (cf. [8]). Similarly, Section 5 is devoted to the proof of Theorem 1.2. The proof is based on the local inversion theorem and the global inversion theorem (see Propositions 5.1 and 5.3). In the final Section 6, by making use of the Leray-Schauder topological degree we prove Theorem 1.3 (Theorem 6.1). 


\section{Local and global inversion theorems}

This section deals with local and global inversions of mappings between Banach spaces which go back to Hadamard in the finite dimensional case and to Cacciopoli and Lévy for general Banach spaces. The presentation here is taken from AmbrosettiProdi [4] and Nirenberg [15] (see also [9]).

\subsection{Local inversion theorem}

Let $X, Y$ be Banach spaces and let $F: X \rightarrow Y$ be a $C^{1}$ map. Namely, the map $F$ is differentiable in $X$ and its Fréchet derivative $D F$ is continuous as a map of $X$ into the Banach space $B(X, Y)$ of bounded (continuous) linear operators from $X$ into $Y$.

A continuous map $F: X \rightarrow Y$ is said to be locally invertible at a point $u^{*}$ of $X$ if there exist an open neighborhood $U$ of $u^{*}$, an open neighborhood $V$ of $F\left(u^{*}\right)$ and a continuous map $G: V \rightarrow U$ such that

$$
\begin{cases}G(F(u))=u & \text { for all } u \in U, \\ F(G(v))=v & \text { for all } v \in V .\end{cases}
$$

The map $G$ is called the local inverse of $F$, and will be denoted by $F^{-1}$.

The local inversion theorem reads as follows (see [4, Chapter 2, Theorem 1.2]):

Theorem 2.1 Let $F: X \rightarrow Y$ be a $C^{1}$ map. If the Fréchet derivative $D F\left(u^{*}\right): X \rightarrow Y$ is continuous and invertible at a point $u^{*} \in X$, then $F$ is locally invertible at $u^{*}$ with $C^{1}$ inverse $F^{-1}$.

\subsection{Global inversion theorem}

Let $M, N$ be metric spaces and let $F: M \rightarrow N$ be a continuous map. The map $F: M \rightarrow$ $N$ is said to be proper if the preimage $F^{-1}(K)$ is compact in $M$ for every compact set $K$ in $N$. We remark that if $F$ is proper, then it maps closed sets in $M$ into closed sets in $N$.

A topological space $T$ is said to be simply connected if it is arcwise connected and if every closed path $\sigma$ in $T$ is homotopic to a constant. Namely, for any given map $\sigma \in C([0,1], T)$ with $\sigma(0)=\sigma(1)$ there exist a map $h \in C([0,1] \times[0,1], T)$ and a point $v \in T$ such that

$$
\begin{cases}h(s, 0)=\sigma(s) & \text { for } 0 \leq s \leq 1 \\ h(s, 1)=v & \text { for } 0 \leq s \leq 1 \\ h(0, t)=h(1, t) & \text { for } 0 \leq t \leq 1\end{cases}
$$

Now we are in position to state the global inversion theorem (see [4, Chapter 3 , Theorem 1.8]):

Theorem 2.2 Let $M$ be an arcwise connected metric space and let $N$ be a simply connected metric space. If a continuous map $F: M \rightarrow N$ is proper and locally invertible on all of $M$, then $F$ is a homeomorphism of $M$ onto $N$. 


\section{The Leray-Schauder degree}

The Leray-Schauder degree is an extension of the Brouwer degree to mappings defined on an infinite dimensional real Banach space. The Brouwer fixed-point theorem asserts that a continuous map $f$ of a closed, bounded convex set $K \subset \mathbf{R}^{n}$ into itself has a fixed point. This is no longer true in infinite dimensions. In infinite-dimensional spaces, we must require more of $f$ than mere continuity (see [15], [7], [23]).

Let $X$ be a real Banach space and let $\Omega$ be a bounded, open subset of $X$ with boundary $\partial \Omega$. A continuous map $f: \Omega \rightarrow X$ is said to be compact if it maps bounded sets in $\Omega$ into relatively compact sets of $X$.

The Leray-Schauder degree $\operatorname{deg}(f, \Omega, p)$ of a compact perturbation $f=I-K$ of the identity map $I$ at a point $p \in X$ and relative to $\Omega$ can be defined by an analogue of the Galerkin approximation procedures, by assuming that

$$
f(x) \neq p \quad \text { on } \partial \Omega .
$$

Similar to the Brouwer degree, the Leray-Schauder degree enjoys some basic properties:

(I) Normalization: If $I: X \rightarrow X$ is the identity map, then we have the assertion

$$
\operatorname{deg}(I, \Omega, p)= \begin{cases}1 & \text { if } p \in \Omega, \\ 0 & \text { if } p \notin \Omega .\end{cases}
$$

(II) Homotopy Invariance: Let $\Omega$ be a bounded open set in $X$. If $K: \bar{\Omega} \times[0,1] \rightarrow X$ is compact and if a point $p \in X$ satisfies the condition

$$
x-K(x, t) \neq p \quad \text { for all } x \in \partial \Omega \text { and } 0 \leq t \leq 1,
$$

then it follows that $\operatorname{deg}(I-K(\cdot, t), \Omega, p)$ is independent of $t$.

(III) Translation Invariance: For each point $p \in X$, we have the formula

$$
\operatorname{deg}(I-K, \Omega, p)=\operatorname{deg}(I-K-p, \Omega, 0) .
$$

(IV) Domain Additivity: If $\Omega_{1}$ and $\Omega_{2}$ are two open subsets in $\Omega$ such that $\Omega_{1} \cap \Omega_{2}=\emptyset$ and satisfy the condition

$$
x-K x \neq p \quad \text { for all } x \in \bar{\Omega} \backslash\left(\Omega_{1} \cup \Omega_{2}\right),
$$

then we have the formula

$$
\operatorname{deg}(I-K, \Omega, p)=\operatorname{deg}\left(I-K, \Omega_{1}, p\right)+\operatorname{deg}\left(I-K, \Omega_{2}, p\right) .
$$

The next theorem is a generalization of Kronecker's existence theorem for the Brouwer degree (see [7, Section 3.4]):

Theorem 3.1 Let $\Omega$ be a bounded open subset of a real Banach space $X$ and let $K: \bar{\Omega} \rightarrow X$ be compact. If $p_{0} \notin(I-K)(\partial \Omega)$ and $\operatorname{deg}\left(I-K, \Omega, p_{0}\right) \neq 0$, then there exists a point $x_{0} \in \Omega$ such that $(I-K) x_{0}=p_{0}$. 


\section{Proof of Theorem 1.1}

This section is devoted to the proof of Theorem 1.1 which is inspired by Ambrosetti-Prodi [4, Theorem 1.13]. The crucial point in the proof is how to verify all the conditions of the global inversion theorem (Theorem 2.2). The proof is divided into three steps.

Step 1: We let

$$
\begin{aligned}
& X=C_{B}^{2+\alpha}(\bar{\Omega})=\left\{u \in C^{2+\alpha}(\bar{\Omega}): B u=a\left(x^{\prime}\right) \frac{\partial u}{\partial v}+b\left(x^{\prime}\right) u=0 \text { on } \partial \Omega\right\}, \\
& Y=C^{\alpha}(\bar{\Omega})
\end{aligned}
$$

and introduce a nonlinear map

$$
F: X \longrightarrow Y
$$

by the formula

$$
F(u)=A u-p(u) \quad \text { for every } u \in X .
$$

Then it is easy to verify the following two assertions:

(1) $D F(u) v=\left(A-p^{\prime}(u)\right) v$ for every $v \in X$.

(2) $F \in C^{1}(X, Y)$.

First, the next proposition verifies the local invertibility of $F$ :

Proposition 4.1 The mapping $F$ is locally invertible on all of $X$.

Proof The proof is based on the local inversion theorem (Theorem 2.1).

(i) First, we prove the Fredholm alternative theorem for the Fréchet derivative $D F(u)$ at a point $u \in X$ :

Lemma 4.1 The index of $D F(u): X \rightarrow Y$ is equal to zero:

$$
\text { ind } D F(u)=\operatorname{dim} N(D F(u))-\operatorname{codim} R(D F(u))=0 .
$$

Proof If we associate with the homogeneous boundary value problem

$$
\begin{cases}A v=f & \text { in } \Omega, \\ B v=0 & \text { on } \partial \Omega\end{cases}
$$

a continuous linear operator $\mathscr{A}$ by the formula

$$
\mathscr{A}=A: X \longrightarrow Y \text {, }
$$

then it follows from an application of [17, Theorem 9.1] with $\varphi:=0$ that the operator $\mathscr{A}$ is a Fredholm operator with index zero:

$$
\text { ind } \mathscr{A}=\operatorname{dim} N(\mathscr{A})-\operatorname{codim} R(\mathscr{A})=0 \text {. }
$$

Moreover, if we let

$$
P(u) v=p^{\prime}(u) v \quad \text { for every } v \in X
$$


then it follows from an application of the Ascoli-Arzelà theorem that the operator

$$
P(u): C^{2+\alpha}(\bar{\Omega}) \longrightarrow C^{\alpha}(\bar{\Omega})
$$

is compact. Therefore, we find that the Fréchet derivative

$$
D F(u)=\mathscr{A}-P(u): X \longrightarrow Y
$$

is a Fredholm operator with index zero, since we have, by assertion (4.1),

$$
\text { ind } D F(u)=\operatorname{ind}(\mathscr{A})=0 \text {. }
$$

The proof of Lemma 4.1 is complete.

(ii) By virtue of Lemma 4.1, it is easy to see that the Fréchet derivative $D F(u)$ at a point $u \in X$ is bijective if and only if $D F(u)$ is injective. Namely, we find that $D F(u)$ is invertible if and only if the linearized eigenvalue problem with the weight $p^{\prime}(u)$

$$
\begin{cases}A v=p^{\prime}(u) v & \text { in } \Omega, \\ B v=0 & \text { on } \partial \Omega\end{cases}
$$

has only the trivial solution. However, we have, by condition (P.3),

$$
p^{\prime}(u(x))<\lambda_{1} \quad \text { for all } x \in \Omega,
$$

and so, by the comparison property ([19, Corollary 3.6]),

$$
\lambda_{1}\left(p^{\prime}(u)\right)>1 \text {. }
$$

This proves that problem (4.2) has only the trivial solution, since 1 is not an eigenvalue of problem (4.2).

(iii) Therefore, we can apply the local inversion theorem (Theorem 2.1) to obtain Proposition 4.1.

The proof of Proposition 4.1 is complete.

Step 2: The next proposition is an essential step in the proof of Theorem 1.1:

Proposition 4.2 The mapping $F: X \rightarrow Y$ is proper.

Proof The proof is divided into three steps.

Step 2-1: Assume that $\left\{h_{n}\right\}$ is an arbitrary bounded sequence in $Y$ such that $F\left(u_{n}\right)=h_{n}$ with $u_{n} \in X$, that is,

$$
\begin{cases}A u_{n}=p\left(u_{n}\right)+h_{n} & \text { in } \Omega, \\ B u_{n}=0 & \text { on } \partial \Omega .\end{cases}
$$

First, we show that the sequence $\left\{u_{n}\right\}$ is bounded from below in the space $C(\bar{\Omega})$. More precisely, we have the following:

Lemma 4.2 There exists a positive constant $c_{1}$ such that

$$
u_{n}(x) \geq-c_{1} \quad \text { on } \bar{\Omega} \text {. }
$$


Proof We consider the following regular (non-degenerate) Robin eigenvalue problem:

$$
\begin{cases}A v=v v & \text { in } \Omega, \\ \frac{\partial v}{\partial v}+\left(\frac{b\left(x^{\prime}\right)}{a\left(x^{\prime}\right)+b\left(x^{\prime}\right)}\right) v=0 & \text { on } \partial \Omega .\end{cases}
$$

Since we have, by conditions (H.1) and (H.2),

$$
\frac{b\left(x^{\prime}\right)}{a\left(x^{\prime}\right)+b\left(x^{\prime}\right)} \not \equiv 0 \quad \text { on } \partial \Omega,
$$

it follows from an application of [18, Theorem 5.1] with

$$
\begin{aligned}
& a\left(x^{\prime}\right):=1, \\
& b\left(x^{\prime}\right):=\frac{b\left(x^{\prime}\right)}{a\left(x^{\prime}\right)+b\left(x^{\prime}\right)}
\end{aligned}
$$

that the first eigenvalue $v_{1}$ of problem (4.5) is positive and algebraically simple, and further that the corresponding eigenfunction $\psi_{1}(x)$ may be chosen to be strictly positive on $\bar{\Omega}$. Namely, we have the assertions

$$
\begin{cases}A \psi_{1}=v_{1} \psi_{1} & \text { in } \Omega \\ \psi_{1}>0 & \text { on } \bar{\Omega} \\ \frac{\partial \psi_{1}}{\partial v}+\left(\frac{b\left(x^{\prime}\right)}{a\left(x^{\prime}\right)+b\left(x^{\prime}\right)}\right) \psi_{1}=0 & \text { on } \partial \Omega\end{cases}
$$

Since the sequence $\left\{h_{n}\right\}$ is bounded in $Y=C^{\alpha}(\bar{\Omega})$, we can find a positive constant $M$ such that

$$
\left\|h_{n}\right\|_{C(\bar{\Omega})} \leq M
$$

If we define a positive constant $C$ by the formula

$$
C=\frac{2 M}{v_{1} \min _{\Omega} \psi_{1}},
$$

then we have, by equations (4.3) and (4.6) and condition (P.1),

$$
\begin{aligned}
A\left(u_{n}+C \psi_{1}\right) & =p\left(u_{n}\right)+h_{n}+C v_{1} \psi_{1} \\
& \geq h_{n}+C v_{1} \psi_{1} \geq h_{n}+C v_{1} \min _{\bar{\Omega}} \psi_{1} \\
& \geq-M+C v_{1} \min _{\bar{\Omega}} \psi_{1}=M \\
& >0 \quad \text { in } \Omega .
\end{aligned}
$$

Moreover, we have, by equations (4.3) and (4.6),

$$
\begin{aligned}
B\left(u_{n}+C \psi_{1}\right) & =C B \psi_{1}=C\left(a\left(x^{\prime}\right) \frac{\partial \psi_{1}}{\partial v}+b\left(x^{\prime}\right) \psi_{1}\right) \\
& =C\left(\frac{b\left(x^{\prime}\right)^{2}}{a\left(x^{\prime}\right)+b\left(x^{\prime}\right)}\right) \psi_{1}
\end{aligned}
$$




$$
\geq 0 \quad \text { on } \partial \Omega \text {. }
$$

Hence it follows that the functions $u_{n}(x)+C \psi_{1}(x)$ satisfy the conditions

$$
\begin{cases}A\left(u_{n}+C \psi_{1}\right)>0 & \text { in } \Omega, \\ B\left(u_{n}+C \psi_{1}\right) \geq 0 & \text { on } \partial \Omega .\end{cases}
$$

Therefore, by applying the maximum principle [18, Theorem 3.7] we obtain that

$$
u_{n}(x)+C \psi_{1}(x) \geq 0 \quad \text { on } \bar{\Omega},
$$

so that

$$
u_{n}(x) \geq-C \psi_{1}(x) \geq-C \max _{\bar{\Omega}} \psi_{1} \quad \text { on } \bar{\Omega} .
$$

This proves the desired estimate (4.4) with

$$
c_{1}=C \max _{\bar{\Omega}} \psi_{1}=\frac{2 M \max _{\bar{\Omega}} \psi_{1}}{v_{1} \min _{\bar{\Omega}} \psi_{1}} .
$$

The proof of Lemma 4.2 is complete.

Step 2-2: Secondly, we show that the sequence $\left\{u_{n}\right\}$ is bounded in the space $C(\bar{\Omega})$. More precisely, we have the following:

Lemma 4.3 There exists a positive constant $c_{2}$ such that

$$
\left\|u_{n}\right\|_{C(\bar{\Omega})} \leq c_{2} .
$$

Proof Assume, to the contrary, that

$$
\left\|u_{n}\right\|_{C(\bar{\Omega})} \longrightarrow \infty \quad \text { as } n \rightarrow \infty
$$

Then, by letting

$$
z_{n}(x)=\frac{u_{n}(x)}{\left\|u_{n}\right\|_{C(\bar{\Omega})}} \quad \text { for all } x \in \bar{\Omega},
$$

we obtain from problem (4.3) that

$$
\begin{cases}A z_{n}=\frac{p\left(u_{n}\right)}{\left\|u_{n}\right\|_{C(\bar{\Omega})}}+\frac{h_{n}}{\left\|u_{n}\right\|_{C(\bar{\Omega})}} & \text { in } \Omega, \\ B z_{n}=0 & \text { on } \partial \Omega .\end{cases}
$$

However, by condition (P.2) we can find a constant $b_{1}$ such that

$$
p(\xi) \leq \gamma \xi+b_{1} \quad \text { for all } \xi \geq-c_{1} .
$$

Hence we have, by inequality (4.4),

$$
p\left(u_{n}(x)\right) \leq \gamma u_{n}(x)+b_{1} \quad \text { for all } x \in \bar{\Omega},
$$

and so

$$
\frac{p\left(u_{n}(x)\right)}{\left\|u_{n}\right\|_{C(\bar{\Omega})}} \leq \gamma z_{n}(x)+\frac{b_{1}}{\left\|u_{n}\right\|_{C(\bar{\Omega})}} \quad \text { for all } x \in \bar{\Omega}
$$


Since $\left\|z_{n}\right\|_{C(\bar{\Omega})}=1$, it follows from inequality (4.12) that the sequence

$$
\frac{p\left(u_{n}\right)}{\left\|u_{n}\right\|_{C(\bar{\Omega})}}
$$

is bounded in the space $C(\bar{\Omega})$. We recall that the sequence $\left\{h_{n}\right\}$ is bounded in the space $C(\bar{\Omega})$.

Therefore, by applying [17, Theorem 9.1] with $\varphi:=0$ for $p>N /(1-\alpha)$ we obtain from problem (4.10) that the sequence

$$
z_{n}=A^{-1}\left(\frac{p\left(u_{n}\right)}{\left\|u_{n}\right\|_{C(\bar{\Omega})}}+\frac{h_{n}}{\left\|u_{n}\right\|_{C(\bar{\Omega})}}\right)
$$

is bounded in the space $C^{1+\alpha}(\bar{\Omega})$. Indeed, it suffices to note that we have, by the Sobolev imbedding theorem (see [1, Theorem 4.12, Part II]),

$$
W^{2, p}(\Omega) \subset C^{2-N / p}(\bar{\Omega}) \subset C^{1+\alpha}(\bar{\Omega}),
$$

for $p>N /(1-\alpha)$. Namely, we have, for some positive constant $C$,

$$
\left\|z_{n}\right\|_{C^{1+\alpha}(\bar{\Omega})} \leq C .
$$

By the Ascoli-Arzelà theorem, we may assume that the sequence $\left\{z_{n}\right\}$ itself converges to some function $z^{*}$ in the space $C^{1}(\bar{\Omega})$ as $n \rightarrow \infty$ :

$$
z_{n} \longrightarrow z^{*} \text { in } C^{1}(\bar{\Omega}) \text { as } n \rightarrow \infty .
$$

We remark that the limit function $z^{*}(x)$ satisfies the condition

$$
\left\|z^{*}\right\|_{C(\bar{\Omega})}=\lim _{n \rightarrow \infty}\left\|z_{n}\right\|_{C(\bar{\Omega})}=1
$$

and also the boundary condition

$$
B z^{*}=\lim _{n \rightarrow \infty} B z_{n}=0 \quad \text { on } \partial \Omega .
$$

Moreover, we have the assertion

$$
z^{*}(x) \geq 0 \quad \text { in } \Omega .
$$

Indeed, if $z^{*}\left(x_{0}\right)<0$ for some point $x_{0} \in \Omega$, then it follows from condition (4.9) that

$$
u_{n}\left(x_{0}\right)=z^{*}\left(x_{0}\right)\left\|u_{n}\right\|_{C(\bar{\Omega})} \longrightarrow-\infty \quad \text { as } n \rightarrow \infty .
$$

This contradicts inequality (4.4).

On the other hand, by multiplying the equation

$$
A z_{n}=\frac{p\left(u_{n}\right)}{\left\|u_{n}\right\|_{C(\bar{\Omega})}}+\frac{h_{n}}{\left\|u_{n}\right\|_{C(\bar{\Omega})}} \quad \text { in } \Omega
$$


by the eigenfunction $\phi_{1}(x)$ and integrating over $\Omega$ we obtain from the selfadjointness of $\mathfrak{A}$ and inequality (4.12) that

$$
\begin{aligned}
\lambda_{1} \int_{\Omega} z_{n}(x) \cdot \phi_{1}(x) d x & =\int_{\Omega} z_{n}(x) \cdot \mathfrak{A} \phi_{1}(x) d x \\
& =\int_{\Omega} \mathfrak{A} z_{n}(x) \cdot \phi_{1}(x) d x \\
& =\int_{\Omega} A z_{n}(x) \cdot \phi_{1}(x) d x \\
& =\int_{\Omega} \frac{p\left(u_{n}\right)}{\left\|u_{n}\right\|_{C(\bar{\Omega})}} \phi_{1}(x) d x+\int_{\Omega} \frac{h_{n}(x) \phi_{1}(x)}{\left\|u_{n}\right\|_{C(\bar{\Omega})}} d x \\
& \leq \gamma \int_{\Omega} z_{n}(x) \phi_{1}(x) d x+\int_{\Omega} \frac{\left(b_{1}+h_{n}(x)\right) \phi_{1}(x)}{\left\|u_{n}\right\|_{C(\bar{\Omega})}} d x
\end{aligned}
$$

Hence, by letting $n \rightarrow \infty$ in inequality (4.16) we obtain from conditions (4.7) and (4.9) and assertion (4.13) that

$$
\lambda_{1} \int_{\Omega} z^{*}(x) \phi_{1}(x) d x \leq \gamma \int_{\Omega} z^{*}(x) \phi_{1}(x) d x .
$$

However, we have, by assertions (4.14) and (4.15),

$$
\int_{\Omega} z^{*}(x) \phi_{1}(x) d x>0
$$

Therefore, it follows from an application of assertions (4.17) and (4.18) that

$$
\lambda_{1} \leq \gamma
$$

This contradicts the condition that $\gamma<\lambda_{1}$ in condition (P.2).

Summing up, we have proved the desired assertion (4.8).

The proof of Lemma 4.3 is complete.

Step 2-3: Thirdly, we show that if $\left\{u_{n}\right\}$ is a sequence in $X=C_{B}^{2+\alpha}(\bar{\Omega})$ such that the sequence

$$
h_{n}=F\left(u_{n}\right)=A u_{n}-p\left(u_{n}\right)
$$

converges to some function $h$ in $Y=C^{\alpha}(\bar{\Omega})$ as $n \rightarrow \infty$, then the sequence $\left\{u_{n}\right\}$ contains a convergent subsequence in $X$. This proves that the mapping $F: X \rightarrow Y$ is proper.

Since the sequence $\left\{u_{n}\right\}$ is bounded in $C(\bar{\Omega})$ as is shown in Lemma 4.3, it follows that the sequence $\left\{p\left(u_{n}\right)+h_{n}\right\}$ is bounded in the space $C(\bar{\Omega})$. Indeed, we have, by inequalities (4.11) and (4.8),

$$
\left\|p\left(u_{n}\right)\right\|_{C(\bar{\Omega})} \leq \gamma\left\|u_{n}\right\|_{C(\bar{\Omega})}+b_{1} \leq \gamma c_{2}+b_{1}
$$

Hence, by applying [17, Theorem 9.1] with $\varphi:=0$ for $p>N /(1-\alpha)$ we obtain from problem (4.3) that the sequence

$$
u_{n}=A^{-1}\left(p\left(u_{n}\right)+h_{n}\right)
$$


is bounded in the space $C^{1+\alpha}(\bar{\Omega})$. Namely, we have, for some positive constant $C^{\prime}$,

$$
\left\|u_{n}\right\|_{C^{1+\alpha}(\bar{\Omega})} \leq C^{\prime} .
$$

Moreover, since $p(\xi)$ is of class $C^{1}$, it follows from inequality (4.20) that the sequence $\left\{p\left(u_{n}\right)+h_{n}\right\}$ is bounded in $C^{\alpha}(\bar{\Omega})$. Therefore, by applying [17, Theorem 9.1] with $\varphi:=0$ we obtain from equation (4.19) that the sequence $\left\{u_{n}\right\}$ is bounded in $C^{2+\alpha}(\bar{\Omega})$. By the Ascoli-Arzelà theorem, we may assume that the sequence $\left\{u_{n}\right\}$ itself converges to some function $u^{*}$ in the space $C^{2}(\bar{\Omega})$ as $n \rightarrow \infty$ :

$$
u_{n} \longrightarrow u^{*} \quad \text { in } C^{2}(\bar{\Omega}) \text { as } n \rightarrow \infty \text {. }
$$

We remark that the limit function $u^{*}(x)$ satisfies the boundary condition

$$
B u^{*}=\lim _{n \rightarrow \infty} B u_{n}=0 \quad \text { on } \partial \Omega .
$$

Moreover, since we have the assertion

$$
A u_{n}=p\left(u_{n}\right)+h_{n} \longrightarrow p\left(u^{*}\right)+h \quad \text { in } C^{\alpha}(\bar{\Omega}) \text { as } n \rightarrow \infty,
$$

it follows from an application of [17, Theorem 9.1] with $\varphi:=0$ that

$$
u_{n}=A^{-1}\left(p\left(u_{n}\right)+h_{n}\right) \longrightarrow A^{-1}\left(p\left(u^{*}\right)+h\right) \quad \text { in } X=C_{B}^{2+\alpha}(\bar{\Omega}) \text { as } n \rightarrow \infty .
$$

In view of assertion (4.21), this proves that

$$
u^{*}=A^{-1}\left(p\left(u^{*}\right)+h\right) \in X,
$$

and further that

$$
u_{n} \longrightarrow u^{*} \quad \text { in } X \text { as } n \rightarrow \infty .
$$

Now the proof of Proposition 4.2 is complete.

Step 3: Finally, by virtue of Propositions 4.1 and 4.2 we can apply the global inversion theorem (Theorem 2.2) to obtain Theorem 1.1.

The proof of Theorem 1.1 is complete.

\section{Proof of Theorem 1.2}

This section is devoted to the proof of Theorem 1.2. The proof is based on the global inversion theorem (Theorem 2.2), and is divided into four steps, just as in Section 4.

Step 1: We let

$$
\begin{aligned}
& X=C_{B}^{2+\alpha}(\bar{\Omega})=\left\{u \in C^{2+\alpha}(\bar{\Omega}): B u=a\left(x^{\prime}\right) \frac{\partial u}{\partial v}+b\left(x^{\prime}\right) u=0 \text { on } \partial \Omega\right\}, \\
& Y=C^{\alpha}(\bar{\Omega}),
\end{aligned}
$$

and introduce a nonlinear map

$$
F: X \longrightarrow Y
$$

by the formula

$$
F(u)=A u-p(u)=A u-a u-q(u) \quad \text { for every } u \in X .
$$

Then it is easy to verify the following two assertions: 
(1) $D F(u) v=\left(A-p^{\prime}(u)\right) v=\left(A-a-q^{\prime}(u)\right) v$ for every $v \in X$.

(2) $F \in C^{1}(X, Y)$.

First, the next proposition verifies the local invertibility of $F$ :

Proposition 5.1 The mapping $F$ is locally invertible on all of $X$.

Proof The proof is based on the local inversion theorem (Theorem 2.1).

(i) First, we prove the Fredholm alternative theorem for the Fréchet derivative $D F(u)$ at a point $u \in X$ :

Lemma 5.1 The index of $D F(u): X \rightarrow Y$ is equal to zero:

$$
\operatorname{ind} D F(u)=\operatorname{dim} N(D F(u))-\operatorname{codim} R(D F(u))=0 .
$$

Proof If we associate with the homogeneous boundary value problem

$$
\begin{cases}A v=f & \text { in } \Omega, \\ B v=0 & \text { on } \partial \Omega\end{cases}
$$

a continuous linear operator $\mathscr{A}$ by the formula

$$
\mathscr{A}=A: X \longrightarrow Y,
$$

then it follows from an application of [17, Theorem 9.1] with $\varphi:=0$ that the operator $\mathscr{A}$ is a Fredholm operator with index zero:

$$
\text { ind } \mathscr{A}=\operatorname{dim} N(\mathscr{A})-\operatorname{codim} R(\mathscr{A})=0 \text {. }
$$

Moreover, if we let

$$
P(u) v=p^{\prime}(u) v=a v+q^{\prime}(u) v \quad \text { for every } v \in X
$$

then it follows from an application of the Ascoli-Arzelà theorem that the operator

$$
P(u): C^{2+\alpha}(\bar{\Omega}) \longrightarrow C^{\alpha}(\bar{\Omega})
$$

is compact. Therefore, we find that the Fréchet derivative

$$
D F(u)=\mathscr{A}-P(u): X \longrightarrow Y
$$

is a Fredholm operator with index zero, since we have, by assertion (5.1),

$$
\text { ind } D F(u)=\text { ind } \mathscr{A}=0 \text {. }
$$

The proof of Lemma 5.1 is complete. 
(ii) By virtue of Lemma 5.1, it is easy to see that the Fréchet derivative $D F(u)$ at a point $u \in X$ is bijective if and only if $D F(u)$ is injective. Namely, we find that $D F(u)$ is invertible if and only if the linearized eigenvalue problem

$$
\begin{cases}A v=p^{\prime}(u) v=a v+q^{\prime}(u) v & \text { in } \Omega, \\ B v=0 & \text { on } \partial \Omega\end{cases}
$$

has only the trivial solution.

If we let

$$
m(x)=p^{\prime}(u) v=a+q^{\prime}(u(x)) \quad \text { for all } x \in \bar{\Omega},
$$

then it follows from condition (Q.3) that

$$
m \in C^{\alpha}(\bar{\Omega})
$$

and further that problem (5.2) can be written as the eigenvalue problem with the weight $m(x)$

$$
\begin{cases}A v=m(x) v & \text { in } \Omega, \\ B v=0 & \text { on } \partial \Omega .\end{cases}
$$

However, we have, by condition (Q.4),

$$
m(x)<\lambda_{1} \text { for all } x \in \bar{\Omega},
$$

or

$$
\lambda_{k}<m(x)<\lambda_{k+1} \quad \text { for all } x \in \bar{\Omega} .
$$

By using the comparison property ([19, Corollary 3.6]), we obtain that

$$
\lambda_{1}(m)>1
$$

or

$$
\lambda_{k}(m)<1<\lambda_{k+1}(m) .
$$

This proves that problem (5.2) has only the trivial solution, since 1 is not an eigenvalue of problem (5.3).

(iii) Therefore, we can apply the local inversion theorem (Theorem 2.1) to obtain Proposition 5.1.

The proof of Proposition 5.1 is complete.

Step 2: The next proposition is an essential step in the proof of Theorem 1.2:

Proposition 5.2 Let $1<p<\infty$. Assume that condition (Q.2) is satisfied. Then there exists a positive constant $C(p, a)$ depending on $p$ and a such that

$$
\|u\|_{W^{2, p}(\Omega)} \leq C(p, a)\|(A-a) u\|_{L^{p}(\Omega)} \quad \text { for all } u \in W_{B}^{2, p}(\Omega) .
$$

Here

$$
W_{B}^{2, p}(\Omega)=\left\{u \in W^{2, p}(\Omega): B u=0 \text { on } \partial \Omega\right\}
$$


Proof Assume, to the contrary, that, for every $n \in \mathbf{N}$ there is a function $u_{n} \in W_{B}^{2, p}(\Omega)$ such that

$$
\left\|u_{n}\right\|_{W^{2, p}(\Omega)}>n\left\|(A-a) u_{n}\right\|_{L^{p}(\Omega)} .
$$

If we let

$$
v_{n}=\frac{u_{n}}{\left\|u_{n}\right\|_{W^{2, p}(\Omega)}} \in W_{B}^{2, p}(\Omega)
$$

then we obtain from equation (5.5) that

$$
\begin{aligned}
& \left\|v_{n}\right\|_{W^{2, p}(\Omega)}=1, \\
& \left\|(A-a) v_{n}\right\|_{L^{p}(\Omega)}<\frac{1}{n} .
\end{aligned}
$$

However, it follows from an application of the Rellich-Kondrachov theorem (see [1, Theorem 6.3]) that the injection $W^{2, p}(\Omega) \rightarrow W^{1, p}(\Omega)$ is compact. By assertion (5.6), we may assume that the sequence $\left\{v_{n}\right\}$ itself converges to some function $v^{*}$ in $W^{1, p}(\Omega)$ as $n \rightarrow \infty:$

$$
v_{n} \longrightarrow v^{*} \quad \text { in } W^{1, p}(\Omega) \text { as } n \rightarrow \infty .
$$

We remark that the limit function $v^{*}(x)$ satisfies the boundary condition

$$
B v^{*}=\lim _{n \rightarrow \infty} B v_{n}=0 \quad \text { on } \partial \Omega .
$$

Furthermore, it follows from inequality (5.7) that

$$
(A-a) v_{n} \longrightarrow 0 \quad \text { in } L^{p}(\Omega) \text { as } n \rightarrow \infty .
$$

Hence we have, by assertion (5.8),

$$
(A-a) v^{*}=\lim _{n \rightarrow \infty}(A-a) v_{n}=0 \quad \text { in } \mathscr{D}^{\prime}(\Omega) .
$$

By combining assertions (5.9) and (5.10), we obtain that

$$
\begin{cases}v^{*} \in W^{1, p}(\Omega), & \\ (A-a) v^{*}=0 & \text { in } \Omega, \\ B v^{*}=0 & \text { on } \partial \Omega .\end{cases}
$$

Hence, it follows from an application of the regularity theorem for problem (5.11) ([17, Theorem 8.2]) that

$$
v^{*} \in W^{2, p}(\Omega) .
$$

On the other hand, by applying [17, Theorem 9.1] with $\varphi:=0$ we can find a positive constant $C$ such that

$$
\|u\|_{W^{2, p}(\Omega)} \leq C\|A u\|_{L^{p}(\Omega)} \quad \text { for all } u \in W_{B}^{2, p}(\Omega) .
$$

By using this inequality with $u:=v_{n}-v^{*}$, we obtain from assertions (5.7), (5.8) and (5.11) that

$$
\left\|v_{n}-v^{*}\right\|_{W^{2, p}(\Omega)}
$$




$$
\begin{aligned}
& \leq C\left\|A\left(v_{n}-v^{*}\right)\right\|_{L^{p}(\Omega)} \\
& =C\left\|(A-a)\left(v_{n}-v^{*}\right)+a\left(v_{n}-v^{*}\right)\right\|_{L^{p}(\Omega)} \\
& \leq C\left\|(A-a) v_{n}\right\|_{L^{p}(\Omega)}+C\left\|(A-a) v^{*}\right\|_{L^{p}(\Omega)}+C|a|\left\|v_{n}-v^{*}\right\|_{L^{p}(\Omega)} \\
& \leq \frac{C}{n}+C|a|\left\|v_{n}-v^{*}\right\|_{L^{p}(\Omega)} \quad \longrightarrow 0 \quad \text { as } n \rightarrow \infty .
\end{aligned}
$$

Hence we have, by assertion (5.6),

$$
\left\|v^{*}\right\|_{W^{2, p}(\Omega)}=\lim _{n \rightarrow \infty}\left\|v_{n}\right\|_{W^{2, p}(\Omega)}=1 .
$$

In particular, it follows that

$$
v^{*}(x) \not \equiv 0 \text { in } \Omega .
$$

Summing up, we have proved that the non-trivial function $v^{*} \in W^{2, p}(\Omega)$ satisfies the conditions

$$
\begin{cases}A v^{*}=a v^{*} & \text { in } \Omega, \\ B v^{*}=0 & \text { on } \partial \Omega .\end{cases}
$$

However, this contradicts condition (Q.2) that the constant $a$ does not coincide with the eigenvalues $\lambda_{k}$ of the operator $\mathfrak{A}$.

The proof of Proposition 5.2 is complete.

Step 3: By using Proposition 5.2, we can prove the following:

Proposition 5.3 The mapping $F: X \rightarrow Y$ is proper.

Proof The proof is divided into two steps.

Step 3-1: Assume that $\left\{h_{n}\right\}$ is an arbitrary bounded sequence in $Y$ such that $F\left(u_{n}\right)=h_{n}$ with $u_{n} \in X$, that is,

$$
\begin{cases}A u_{n}-a u_{n}-q\left(u_{n}\right)=h_{n} & \text { in } \Omega, \\ B u_{n}=0 & \text { on } \partial \Omega .\end{cases}
$$

First, we show that the sequence $\left\{u_{n}\right\}$ is bounded in the space $Y=C^{\alpha}(\bar{\Omega})$. Namely, we have the following:

Lemma 5.2 There exists a positive constant $c_{1}$ such that

$$
\left\|u_{n}\right\|_{Y} \leq c_{1} .
$$

Proof Assume, to the contrary, that

$$
\left\|u_{n}\right\|_{Y} \longrightarrow \infty \quad \text { as } n \rightarrow \infty
$$

If we let

$$
z_{n}(x)=\frac{u_{n}(x)}{\left\|u_{n}\right\|_{Y}} \quad \text { for all } x \in \bar{\Omega},
$$


then we obtain from problem (5.12) that

$$
\begin{cases}(A-a) z_{n}=\frac{q\left(u_{n}\right)}{\left\|u_{n}\right\|_{Y}}+\frac{h_{n}}{\left\|u_{n}\right\|_{Y}} & \text { in } \Omega, \\ B z_{n}=0 & \text { on } \partial \Omega .\end{cases}
$$

However, it follows from condition (Q.3) that the sequence

$$
U_{n}=\frac{q\left(u_{n}\right)}{\left\|u_{n}\right\|_{Y}}+\frac{h_{n}}{\left\|u_{n}\right\|_{Y}}
$$

is bounded in the space $C(\bar{\Omega})$, since the functions $q\left(u_{n}(x)\right)$ and $h_{n}(x)$ are bounded in $C(\bar{\Omega})$. More precisely, we have, by assertion (5.14),

$$
U_{n} \longrightarrow 0 \quad \text { in } C(\bar{\Omega}) \text { as } n \rightarrow \infty .
$$

By applying inequality (5.4) with $u:=z_{n}$ for $p>N /(1-\alpha)$, we obtain from the equation

$$
(A-a) z_{n}=U_{n} \quad \text { in } \Omega
$$

that the sequence $\left\{z_{n}\right\}$ is bounded in the space $C^{1+\alpha}(\bar{\Omega})$. Namely, we have, for some positive constant $C$,

$$
\left\|z_{n}\right\|_{C^{1+\alpha}(\bar{\Omega})} \leq C
$$

Indeed, it follows from an application of the Sobolev imbedding theorem (see [1, Theorem 4.12, Part II]) that we have the imbedding

$$
W^{2, p}(\Omega) \subset C^{2-N / p}(\bar{\Omega}) \subset C^{1+\alpha}(\bar{\Omega}),
$$

for $p>N /(1-\alpha)$.

Hence, by the Ascoli-Arzelà theorem we may assume that the sequence $\left\{z_{n}\right\}$ itself converges to some function $z^{*}$ in the space $C^{1}(\bar{\Omega})$ as $n \rightarrow \infty$ :

$$
z_{n} \longrightarrow z^{*} \text { in } C^{1}(\bar{\Omega}) \text { as } n \rightarrow \infty \text {. }
$$

We remark that the limit function $z^{*}(x)$ satisfies the condition

$$
\left\|z^{*}\right\|_{Y}=\lim _{n \rightarrow \infty}\left\|z_{n}\right\|_{Y}=1
$$

and also the boundary condition

$$
B z^{*}=\lim _{n \rightarrow \infty} B z_{n}=0 \quad \text { on } \partial \Omega
$$

In particular, it follows that $z^{*}(x) \not \equiv 0$ in $\Omega$.

Therefore, by passing to the limit in equation (5.16) we obtain from assertions (5.15), (5.17) and (5.18) that

$$
\begin{cases}(A-a) z^{*}=0 & \text { in } \Omega \\ B z^{*}=0 & \text { on } \partial \Omega .\end{cases}
$$


Moreover, it follows from an application of the regularity theorem for problem (5.19) ([17, Theorem 8.2]) that

$$
z^{*} \in C^{2+\alpha}(\bar{\Omega}) .
$$

Summing up, we have proved that the non-trivial function $z^{*} \in C^{2+\alpha}(\bar{\Omega})$ satisfies the conditions

$$
\begin{cases}A z^{*}=a z^{*} & \text { in } \Omega, \\ B z^{*}=0 & \text { on } \partial \Omega .\end{cases}
$$

However, this contradicts condition (Q.2) that the constant $a$ does not coincide with the eigenvalues $\lambda_{k}$ of the operator $\mathfrak{A}$.

The proof of Lemma 5.2 is complete.

Step 3-2: Secondly, we show that if $\left\{u_{n}\right\}$ is a sequence in $X$ such that the sequence

$$
F\left(u_{n}\right)=A u_{n}-a u_{n}-q\left(u_{n}\right)=h_{n}
$$

converges to some function $h$ in $X$ as $n \rightarrow \infty$, then the sequence $\left\{u_{n}\right\}$ contains a convergent subsequence in $X$. This proves that the mapping $F: X \rightarrow Y$ is proper.

By inequality (5.13), it is easy to verify that the sequence

$$
\theta_{n}=a u_{n}+q\left(u_{n}\right)+h_{n}
$$

is bounded in the space $Y=C^{\alpha}(\bar{\Omega})$. Hence, by applying [17, Theorem 9.1] with $\varphi:=0$ we obtain from problem (5.12) that the sequence

$$
u_{n}=A^{-1}\left(\theta_{n}\right)
$$

is bounded in the space $C^{2+\alpha}(\bar{\Omega})$. Namely, we have, for some positive constant $C^{\prime}$,

$$
\left\|u_{n}\right\|_{C^{2+\alpha}(\bar{\Omega})} \leq C^{\prime}
$$

By the Ascoli-Arzelà theorem, we may assume that the sequence $\left\{u_{n}\right\}$ itself converges to some function $u^{*}$ in the space $C^{2}(\bar{\Omega})$ as $n \rightarrow \infty$ :

$$
u_{n} \longrightarrow u^{*} \quad \text { in } C^{2}(\bar{\Omega}) \text { as } n \rightarrow \infty \text {. }
$$

We remark that the limit function $u^{*}(x)$ satisfies the boundary condition

$$
B u^{*}=\lim _{n \rightarrow \infty} B u_{n}=0 \quad \text { on } \partial \Omega .
$$

Moreover, since we have the assertion

$$
A u_{n}=\theta_{n}=a u_{n}+q\left(u_{n}\right)+h_{n} \longrightarrow a u^{*}+q\left(u^{*}\right)+h \quad \text { in } Y=C^{\alpha}(\bar{\Omega}) \text { as } n \rightarrow \infty,
$$

it follows from an application of [17, Theorem 9.1] with $\varphi:=0$ that

$$
\begin{aligned}
& u_{n}=A^{-1}\left(\theta_{n}\right)=A^{-1}\left(a u_{n}+q\left(u_{n}\right)+h_{n}\right) \\
& \longrightarrow A^{-1}\left(a u^{*}+q\left(u^{*}\right)+h\right) \quad \text { in } X=C_{B}^{2+\alpha}(\bar{\Omega}) \text { as } n \rightarrow \infty .
\end{aligned}
$$

In view of assertion (5.20), this proves that

$$
u^{*}=A^{-1}\left(a u^{*}+q\left(u^{*}\right)+h\right) \in X,
$$

and further that

$$
u_{n} \longrightarrow u^{*} \quad \text { in } X \text { as } n \rightarrow \infty .
$$

Now the proof of Proposition 5.3 is complete. 
Step 4: Finally, by virtue of Propositions 5.1 and 5.3 we can apply the global inversion theorem (Theorem 2.2) to obtain Theorem 1.2.

The proof of Theorem 1.2 is complete.

\section{Proof of Theorem 1.3}

This section is devoted to the proof of Theorem 1.3. The next existence theorem proves Theorem 1.3:

Theorem 6.1 Assume that the nonlinear term $q(\xi)$ satisfies condition $(Q .5)$. Then the semilinear boundary value problem

$$
\begin{cases}A u-a u-q(u)=h & \text { in } \Omega, \\ B u=a\left(x^{\prime}\right) \frac{\partial u}{\partial v}+b\left(x^{\prime}\right) u=0 & \text { on } \partial \Omega\end{cases}
$$

has a solution $u \in C^{2+\alpha}(\bar{\Omega})$ for any $h \in C^{\alpha}(\bar{\Omega})$.

Proof The proof of Theorem 6.1 is based on Theorem 3.1, and is divided into four steps.

Step 1: First, we let

$$
\begin{aligned}
& X=C_{B}^{2+\alpha}(\bar{\Omega})=\left\{u \in C^{2+\alpha}(\bar{\Omega}): B u=0 \quad \text { on } \partial \Omega\right\}, \\
& Y=C^{\alpha}(\bar{\Omega}) .
\end{aligned}
$$

Then we have the following:

Lemma 6.1 If $u \in C^{2+\alpha}(\bar{\Omega})$ is a solution of problem (6.1), then there exists a positive constant $c$ such that

$$
\|u\|_{Y} \leq c .
$$

Proof Assume, to the contrary, that, for every $n \in \mathbf{N}$ there is a function $u_{n} \in X$ such that

$$
\begin{cases}A u_{n}-a u_{n}-q\left(u_{n}\right)=h & \text { in } \Omega, \\ B u_{n}=0 & \text { on } \partial \Omega,\end{cases}
$$

and that

$$
\left\|u_{n}\right\|_{Y}>n
$$

If we let

$$
z_{n}(x)=\frac{u_{n}(x)}{\left\|u_{n}\right\|_{Y}} \in X
$$

then we obtain from problem (6.2) that

$$
\begin{cases}(A-a) z_{n}=\frac{q\left(u_{n}\right)}{\left\|u_{n}\right\|_{Y}}+\frac{h}{\left\|u_{n}\right\|_{Y}} & \text { in } \Omega, \\ B z_{n}=0 & \text { on } \partial \Omega .\end{cases}
$$


However, since $q(\xi)$ is bounded on $\mathbf{R}$, it follows from condition (6.3) that the sequence

$$
U_{n}=\frac{q\left(u_{n}\right)}{\left\|u_{n}\right\|_{Y}}+\frac{h}{\left\|u_{n}\right\|_{Y}}
$$

converges to zero in $C(\bar{\Omega})$ as $n \rightarrow \infty$ :

$$
U_{n} \longrightarrow 0 \quad \text { in } C(\bar{\Omega}) \text { as } n \rightarrow \infty .
$$

Therefore, by applying inequality (5.4) with $u:=z_{n}$ for $p>N /(1-\alpha)$ we obtain from problem (6.4) that the sequence $\left\{z_{n}\right\}$ is bounded in $C^{1+\alpha}(\bar{\Omega})$. Hence, by the Ascoli-Arzelà theorem we may assume that the sequence $\left\{z_{n}\right\}$ itself converges to some function $z^{*}$ in the space $C^{1}(\bar{\Omega})$ as $n \rightarrow \infty$ :

$$
z_{n} \longrightarrow z^{*} \quad \text { in } C^{1}(\bar{\Omega}) \text { as } n \rightarrow \infty \text {. }
$$

By passing to the limit in problem (6.4), we obtain from assertions (6.5) and (6.6) that the limit function $z^{*}(x)$ satisfies the conditions

$$
\begin{cases}(A-a) z^{*}=0 & \text { in } \Omega, \\ B z^{*}=0 & \text { on } \partial \Omega\end{cases}
$$

and also

$$
\left\|z^{*}\right\|_{Y}=\lim _{n \rightarrow \infty}\left\|z_{n}\right\|_{Y}=1 .
$$

Furthermore, it follows from an application of the regularity theorem for problem (6.7) ([17, Theorem 8.2]) that

$$
z^{*} \in C^{2+\alpha}(\bar{\Omega}) \text {. }
$$

Summing up, we have proved that the non-trivial function $z^{*} \in C^{2+\alpha}(\bar{\Omega})$ satisfies the conditions

$$
\begin{cases}A z^{*}=a z^{*} & \text { in } \Omega, \\ B z^{*}=0 & \text { on } \partial \Omega .\end{cases}
$$

However, this contradicts condition (Q.2) that the constant $a$ does not coincide with the eigenvalues $\lambda_{k}$ of the operator $\mathfrak{A}$.

The proof of Lemma 6.1 is complete.

Step 2: Secondly, we consider the following family of semilinear elliptic boundary value problems:

$$
\begin{cases}A u-a u-t q(u)=h & \text { in } \Omega, \\ B u=0 & \text { on } \partial \Omega,\end{cases}
$$

where $0 \leq t \leq 1$. We remark that problem (6.8) coincides with the original problem (6.1) when $t=1$.

It follows from an application of Proposition 5.2 that the inverse $(-A+a I)^{-1}$ exists for $a \neq \lambda_{k}, k \geq 1$. Hence we can rewrite problem (6.8) in the operator equation form

$$
u-t(A-a I)^{-1}(q(u))=(A-a I)^{-1} h \quad \text { in } X .
$$


If we let

$$
\left\{\begin{array}{l}
K(u, t)=t(A-a I)^{-1}(q(u)) \quad \text { for all } u \in X \text { and } 0 \leq t \leq 1, \\
g=(A-a I)^{-1} h \in X
\end{array}\right.
$$

then we obtain that equation (6.9) is equivalent to the following operator equation:

$$
u-K(u, t)=g \quad \text { in } X .
$$

The next proposition is an essential step in the proof of Theorem 6.1:

Proposition 6.1 The mapping

$$
K: X \times[0,1] \longrightarrow X
$$

is compact.

Proof Assume that $\left\{\left(u_{n}, t_{n}\right)\right\}$ is an arbitrary bounded sequence in $X \times[0,1]$. Then, by the Ascoli-Arzelà theorem we may assume that the sequence $\left\{u_{n}\right\}$ itself converges to some function $u^{*}$ in the space $C^{2}(\bar{\Omega})$ as $n \rightarrow \infty$ and further that the sequence $\left\{t_{n}\right\}$ itself converges to some point $t^{*}$ in $[0,1]$ as $n \rightarrow \infty$.

Since $q(\xi)$ is bounded and Lipschitz continuous on $\mathbf{R}$, we have the following two assertions:

(i) $q\left(u_{n}\right) \rightarrow q\left(u^{*}\right)$ in $C(\bar{\Omega})$.

(ii) The sequence $\left\{q\left(u_{n}\right)-q\left(u^{*}\right)\right\}$ is bounded in the space $C^{0,1}(\bar{\Omega})$ of Lipschitz continuous functions on $\bar{\Omega}$.

However, it follows from an application of the Ascoli-Arzelà theorem that the injection $C^{0,1}(\bar{\Omega}) \rightarrow C^{\alpha}(\bar{\Omega})$ is compact for $0<\alpha<1$. Hence we find from assertions (i) and (ii) that the sequence $\left\{q\left(u_{n}\right)-q\left(u^{*}\right)\right\}$ accumulates only at 0 in the space $C^{\alpha}(\bar{\Omega})$. This proves that $q\left(u_{n}\right) \rightarrow q\left(u^{*}\right)$ in $Y=C^{\alpha}(\bar{\Omega})$ as $n \rightarrow \infty$.

On the other hand, by using [17, Theorem 9.1] with $\varphi:=0$ in the proof of Proposition 5.2 we obtain that the inverse $(A-a I)^{-1}: Y \rightarrow X$ is bounded:

$$
\left\|(A-a I)^{-1}\right\|=\sup _{\substack{v \in Y \\ v \neq 0}} \frac{\left\|(A-a I)^{-1} v\right\|_{X}}{\|v\|_{Y}}<\infty .
$$

Therefore, it follows that the sequence

$$
K\left(u_{n}, t_{n}\right)=t_{n}(A-a I)^{-1}\left(q\left(u_{n}\right)\right)
$$

converges to the function

$$
K\left(u^{*}, t^{*}\right)=t^{*}(A-a I)^{-1}\left(q\left(u^{*}\right)\right)
$$

in $X$ as $n \rightarrow \infty$.

Summing up, we have proved that the mapping $K: X \times[0,1] \rightarrow X$ is compact.

The proof of Proposition 6.1 is complete.

Step 3: Thirdly, we have the following: 
Proposition 6.2 For the function $g=(A-a I)^{-1} h \in X$ with $h \in Y$, the set

$$
S=\{u \in X: u-K(u, t)=g \text { for some } t \in[0,1]\}
$$

is bounded in X. Namely, there exists a positive constant $M$, independent of $t$, such that

$$
\|u\|_{X} \leq M \quad \text { for all } u \in S .
$$

Proof By arguing just as in the proof of Lemma 6.1, we obtain that the set $S$ is bounded in $Y$. Namely, there exists a positive constant $M_{0}$, independent of $t$, such that

$$
\|u\|_{Y} \leq M_{0} \quad \text { for all } u \in S .
$$

On the other hand, since $q(\xi)$ is bounded and Lipschitz continuous on $\mathbf{R}$, it is easy to see that

$$
\|q(u)\|_{Y} \leq \sup _{\xi \in \mathbf{R}}|q(\xi)|+L\|u\|_{Y}
$$

where $L$ is a Lipschitz constant for the function $q(\xi)$.

Hence we have, by assertion (6.10) and inequalities (6.13) and (6.12),

$$
\begin{aligned}
\|K(u, t)\|_{X} & =t\left\|(A-a I)^{-1}(q(u))\right\|_{X} \leq t\left\|(A-a I)^{-1}\right\| \cdot\|q(u)\|_{Y} \\
& \leq\left\|(A-a I)^{-1}\right\|\left(\sup _{\xi \in \mathbf{R}}|q(\xi)|+L\|u\|_{Y}\right) \\
& \leq\left\|(A-a I)^{-1}\right\|\left(\sup _{\xi \in \mathbf{R}}|q(\xi)|+L M_{0}\right) \quad \text { for all } u \in S .
\end{aligned}
$$

Therefore, we obtain the inequality

$$
\begin{aligned}
\|u\|_{X} & =\|K(u, t)+g\|_{X} \leq\|K(u, t)\|_{X}+\|g\|_{X} \\
& \leq\left\|(A-a I)^{-1}\right\|\left(\sup _{\xi \in \mathbf{R}}|q(\xi)|+L M_{0}\right)+\|g\|_{X} \quad \text { for all } u \in S .
\end{aligned}
$$

This proves the desired inequality (6.11) with

$$
M=\left\|(A-a I)^{-1}\right\|\left(\sup _{\xi \in \mathbf{R}}|q(\xi)|+L M_{0}\right)+\|g\|_{X} .
$$

The proof of Proposition 6.2 is complete.

Step 4: Finally, if we let

$$
B(0,2 M)=\left\{u \in X:\|u\|_{X}<2 M\right\},
$$

then we obtain from Proposition 6.2 that

$$
u-K(u, t) \neq g \quad \text { for all }\|u\|_{X}=2 M \text { and } 0 \leq t \leq 1 .
$$


By virtue of Proposition 6.1, it follows from the homotopy invariance and normalization of the Leray-Schauder degree that

$$
\begin{aligned}
\operatorname{deg}\left(I-(A-a I)^{-1} q(\cdot), B(0,2 M), g\right) & =\operatorname{deg}(I-K(\cdot, 1), B(0,2 M), g) \\
& =\operatorname{deg}(I-K(\cdot, 0), B(0,2 M), g) \\
& =\operatorname{deg}(I, B(0,2 M), g) \\
& =1
\end{aligned}
$$

Therefore, by applying Theorem 3.1 with

$$
\begin{aligned}
& X:=C^{2+\alpha}(\bar{\Omega}), \quad \Omega:=B(0,2 M), \\
& K:=K(\cdot, 1)=(A-a I)^{-1} q(\cdot), \quad p_{0}:=g,
\end{aligned}
$$

we obtain from assertions (6.14) and (6.15) that the operator equation

$$
u-(A-a I)^{-1}(q(u))=g=(A-a I)^{-1} h
$$

has a solution $u$ in the open ball $B(0,2 M)$ of $X$.

Summing up, we have proved that problem (6.1) has a solution $u \in C^{2+\alpha}(\bar{\Omega})$ for any $h \in C^{\alpha}(\bar{\Omega})$.

Now the proof of Theorem 6.1 is complete.

Acknowledgements The author is grateful to the referee for many valuable suggestions and some additional references, which helped him to improve the presentation of the present paper. Especially, Theorem 1.3 was suggested by the referee. This research is partially supported by Grant-in-Aid for General Scientific Research (No. 19540162), Ministry of Education, Culture, Sports, Science and Technology, Japan.

\section{References}

1. Adams, R.A., Fournier, J.J.F.: Sobolev spaces, second edition. Academic Press, Amsterdam Heidelberg New York Oxford (2003)

2. Amann, H.: Fixed point equations and nonlinear eigenvalue problems in ordered Banach spaces. SIAM Rev. 18, 620-709 (1976)

3. Ambrosetti, A., Prodi, G.: On the inversion of some differentiable mappings with singularities between Banach spaces. Ann. Mat. Pura Appl. 93, 231-247 (1972)

4. Ambrosetti, A., Prodi, G.: A primer of nonlinear analysis. Cambridge University Press, Cambridge (1993)

5. Berger, M.S., Podolak, E.: On the solutions of a nonlinear Dirichlet problem. Indiana Univ. Math. J. 24, 837-846 (1974/75)

6. Castro, A., Lazer, A.C.: Critical point theory and the number of solutions of a nonlinear Dirichlet problem. Ann. Mat. Pura Appl. 120, 113-137 (1979)

7. Chang, K.C.: Methods in nonlinear analysis. Springer-Verlag, Berlin Heidelberg New York (2005)

8. de Figueiredo, D.G.: Positive solutions of semilinear elliptic problems. In: Differential equations, Lecture Notes in Mathematics, No. 957, Springer-Verlag, Berlin Heidelberg New York (1982), 34-87

9. Dieudonné, J.: Foundations of modern analysis. Academic Press, New York London (1969)

10. Dolph, C.L.: Nonlinear integral equations of the Hammerstein-type. Trans. Amer. Math. Soc. 66, 289-307 (1949)

11. Hammerstein, A.: Nichtlineare Integralgleichungen nebst Anwendungen. Acta Math. 54, 117-176 (1930)

12. Hörmander, L.: The analysis of linear partial differential operators III. Springer-Verlag, Berlin Heidelberg New York Tokyo (1985) 
13. Leray, J., Schauder, J.: Topologie et équations fonctionelles. Ann. Sci. École Norm. Sup. 51, 45-78 (1934)

14. Mawhin, J.: Topological degree methods in nonlinear boundary value problems. CBMS Regional Conference Series in Mathematics, No. 40, American Mathematical Society, Providence, Rhode Island (1979)

15. Nirenberg, L.: Topics in nonlinear functional analysis. Courant Institute of Mathematical Sciences, New York University, New York (1974)

16. Runst, T., Sickel, W.: Sobolev spaces of fractional order, Nemytskij operators, and nonlinear partial differential equations. Walter de Gruyter, Berlin New York (1996)

17. Taira, K.: Semigroups, boundary value problems and Markov processes. Springer-Verlag, Berlin Heidelberg New York (2004)

18. Taira, K.: Degenerate elliptic boundary value problems with indefinite weights. Mediterranean J. Math. 5, 133-162 (2008)

19. Taira, K.: Degenerate elliptic boundary value problems with asymmetric nonlinearity. J. Math. Soc. Japan 62, 431-465 (2010)

20. Taira, K.: Semilinear degenerate elliptic boundary value problems at resonance. Ann. Univ. Ferrara 56, 369-392 (2010)

21. Taira, K.: Multiple solutions of semilinear degenerate elliptic boundary value problems. Math. Nachr. 284, 105-123 (2011)

22. Thom, R.: Les singularités des applications différentiables. Ann. Inst. Fourier (Grenoble) 6, 43-87 (1955-1956)

23. Zeidler, E.: Nonlinear functional analysis and its applications I. Springer-Verlag, New York Berlin Heidelberg (1986) 